\title{
Representation Dependence of Superficial Degree of Divergences in Quantum Field Theory
}

\author{
Abouzeid. M. Shalaby* \\ Physics Department, Faculty of Science, Mansoura University, Egypt
}

\begin{abstract}
In this work, we investigate a very important but unstressed result in the work of Carl M. Bender, Jun-Hua Chen, and Kimball A. Milton ( J.Phys.A39:1657-1668, 2006). In this article, Bender et.al have calculated the vacuum energy of the $i \phi^{3}$ scalar field theory and its Hermitian equivalent theory up to $g^{4}$ order of calculations. While all the Feynman diagrams of the $i \phi^{3}$ theory are finite in $0+1$ space-time dimensions, some of the corresponding Feynman diagrams in the equivalent Hermitian theory are divergent. In this work, we show that the divergences in the Hermitian theory originate from superrenormalizable, renormalizable and non-renormalizable terms in the interaction Hamiltonian even though the calculations are carried out in the $0+1$ spacetime dimensions. Relying on this interesting result, we raise the question, is the superficial degree of divergence of a theory is representation dependent? To answer this question, we introduce and study a class of non-Hermitian quantum field theories characterized by a field derivative interaction Hamiltonian. We showed that the class is physically acceptable by finding the corresponding class of metric operators in a closed form. We realized that the obtained equivalent Hermitian and the introduced non-Hermitian representations have coupling constants of different mass dimensions which may be considered as a clue for the possibility of considering non-Renormalizability of a field theory as a non-genuine problem. Besides, the metric operator is supposed to disappear from path integral calculations which means that physical amplitudes can be fully obtained in the simpler non-Hermitian representation.
\end{abstract}

PACS numbers: 03.65.Ca, 11.90.+t, 11.10.Lm, 11.10.Gh

Keywords: Pseudo-Hermitian Hamiltonians, Metric Operators, Non-Hermitian models, Non-Renormalizable theories, PT-Symmetric theories.

\footnotetext{
* E-mail:amshalab@mans.edu.eg
} 
Two main reasons prevent a theory from playing a role in the description of matter interactions. In a historical order, the first reason is non-Hermiticity of Hamiltonian models which for long time prevented any try to take non-Hermitian theories into account in the search for a suitable mathematical description that mimic known features of nature. For instance, in the standard model for particle interactions one had to resort to a non-Abelean theory of high group structures to obtain the asymptotic freedom property (QCD). Recently, an idea back to Symanzik has been stressed and it is now well known that a simple Abelean theory can exhibit asymptotic freedom [1 5]. Moreover, also in the standard model, Hemriticity obliged us to employ a spontaneous symmetry breaking algorithm using only Hermitian scalar field theory which leaded to the famous Hierarchy problem. In Ref. [6], we showed that the behavior of a non-Hermitian scalar field theory at high energy scales is secure rather than the Hermitian scalar field theory for which the mass parameter and all the dimensionfull parameters blow up to unacceptable values and thus leading to the Hierarchy and the cosmological constant problems [16]. Rather than these interesting features of a non-Hermitian theory, the techniques used by the field of pseudo-Hermitian theories can be employed to solve some problems in Physics. For instance, in Ref. [7], we showed that the algorithm can be used to cure the ghost states in the Lee-Wick standard model. In fact, this trend has been initiated by Bender et.al in Refs. [8, 9].

The renormalizability of a theory is the second reason that prevents a theory from playing a role in describing a physical system. For instance, the particle physics community celebrated the discovery of the Weinberg-Salam model for its renormalizability and was ready to replace the non-Renormalizable Fermi model introduced to describe Weak interactions. Another famous problem is the unification of the four forces which up till now is far from reaching a suitable treatment due to the non-Renormalizability of the theory describing gravitational interactions. Besides, in the early universe studies, one needs to resort to a theory which is capable of making a strong first order phase transition to account for matterantimatter asymmetry in the universe [10]. A well known theory that can have such feature is the non-renormalizable $\phi^{6}$ scalar field theory [11].

The first reason mentioned above is no longer a holy belief and one can show that there exists an infinite number of Hamiltonians which are neither Hermitian nor $\mathcal{P} \mathcal{T}$-symmetric and have real spectra as well. On the other hand, normalizability have not been stressed in the sense that there are no known technique by which one can get rid of it. In this work 
we argue that a question of the form "is non-renormalizability of a theory is a technical or a conceptual problem?" is legal. Also, one may ask if it is technical then what is the calculational algorithm that can be used to get rid of it? However, before trying to answer this question, we refer to an unstressed but interesting result in Ref. [12]. In this article, the authors have obtained the vacuum energy of the $\mathcal{P} \mathcal{T}$-symmetric $\left(i \phi^{3}\right)$ scalar field theory which ought to be the same as the vacuum energy of the corresponding equivalent Hermitian theory. Although the $\mathcal{P} \mathcal{T}$-symmetric $\left(i \phi^{3}\right)$ is finite in $0+1$ space time dimensions, the authors found that the Hermitian theory suffers from the existence of divergences. This interesting result pushed us to ask the above question because the results in Ref. [12] show the equivalence between finite theory and a theory with divergences. In fact, Bender et.al showed that the finite non-Hermitian theory described by the Hamiltonian density of the form;

$$
H=\frac{\pi^{2}}{2}+\frac{1}{2}(\nabla \phi)^{2}+\frac{1}{2} m^{2} \phi^{2}+i g \phi^{3},
$$

in $0+1$ dimensions of the space-time is equivalent to the divergent Hermitian Hamiltonian of the form;

$$
\begin{aligned}
h & =\frac{\pi^{2}}{2}+\frac{1}{2} m^{2} \phi^{2}+\left(\frac{3 g^{2}}{2 m^{2}} \phi^{4}+3 \frac{g^{2}}{m^{4}} S_{2,2}-\frac{g^{2}}{2 m^{4}}\right) \\
& +\left(-\frac{7}{6} \frac{g^{4}}{m^{6}} \phi^{6}-\frac{51}{2} \frac{g^{4}}{m^{8}} S_{2,4}-36 \frac{g^{4}}{m^{10}} S_{4,2}+2 \frac{g^{4}}{m^{12}} \pi^{6}+\frac{15 g^{4}}{2 m^{8}} \phi^{2}+27 \frac{g^{4}}{m^{10}} \pi^{2}\right),
\end{aligned}
$$

where we set the mass parameter $m$ explicitly and the operator $(\nabla \phi)^{2}$ has been dropped out because it is certainly zero in $0+1$ dimensions. Also, the symbol $S_{i, j}$ represents a symmetric combination of $j$ factors of the field variable $\phi$ and $i$ factors of its conjugate field $\pi$. To understand well the source of the divergent diagrams resulted in the Hamiltonian in Eq.(1), let us regroup the different terms in $h$ as;

$$
\begin{aligned}
h & =h_{0}+h_{f}+h_{s r}+h_{r}+h_{n r}, \\
h_{0} & =\frac{\pi^{2}}{2}+\frac{1}{2} m^{2} \phi^{2} \\
h_{f} & =\frac{3 g^{2}}{2 m^{2}} \phi^{4}-\frac{7}{6} \frac{g^{4}}{m^{6}} \phi^{6}-\frac{g^{2}}{2 m^{4}}, \\
h_{s r} & =\frac{15 g^{4}}{2 m^{8}} \phi^{2}+3 \frac{g^{2}}{m^{4}} S_{2,2}-\frac{51}{2} \frac{g^{4}}{m^{8}} S_{2,4}, \\
h_{r} & =-36 \frac{g^{4}}{m^{10}} S_{4,2}+27 \frac{g^{4}}{m^{10}} \pi^{2}, \\
h_{n r} & =2 \frac{g^{4}}{m^{12}} \pi^{6}
\end{aligned}
$$


where $h_{0}$ is the free Hamiltonian. Since the superficial degree of divergence of a theory depends crucially on the mass dimension of the coupling [13], we realize that, in $0+1$ spacetime dimensions, the couplings in $h_{f}$ have mass dimension greater than 2 and thus any diagram generated from the contractions of the fields in $h_{f}$ is finite. For $h_{s r}$, the couplings dimensions are positive but less than or equal 2 which means that this term represents a super renorlaizable interaction Hamiltonian and thus one might find finite as well as infinite Feynman diagrams generated by $h_{s r}$. Similarly, $h_{r}$ is renormalizable (has a dimensionless coupling) while $h_{n r}$ is non-Renormalizable ( has a coupling of negative mass dimension) interaction Hamiltonians even in $0+1$ space-time dimensions (quantum mechanics). In view of these analysis, our important realization that the results in Ref. [12] show an equivalence between finite (non-Hermitian) and infinite (Hermitian) theories leaded us to believe that the superficial degree of divergence is representation dependent.

For more elaboration of the possible equivalence between two theories of different superficial degrees of divergence, in this work, we introduce and study a class of non-Hermitian field theories for which the corresponding equivalent Hermitian Hamiltonians are obtained in a closed form. The Hermitian class of Hamiltonians has superficial degree of divergences which are different from those of the corresponding non-Hermitian class of Hamiltonians. By this work, we want to spread the message that one can play on the dimension (in terms of mass) of the coupling in a theory and gets an equivalent theory with a coupling of different mass dimension.

To start, let us consider the Hamiltonian model of the form

$$
\begin{aligned}
H & =\int d x^{3}\left(\begin{array}{c}
\frac{\pi^{2}(x)}{2}+\frac{1}{2}(\nabla \phi(x))^{2}+\frac{m^{2}}{2} \phi^{2}(x) \\
+\frac{\lambda}{4 !} \phi^{4}(x)+\frac{i \xi}{\sqrt{6 !}}\left\{\phi^{3}(x), \pi(x)\right\}
\end{array}\right), \\
& =H_{0}+\epsilon H_{I}, \\
H_{0} & =\int d x^{3}\left(\frac{\pi^{2}(x)}{2}+\frac{1}{2}(\nabla \phi(x))^{2}+\frac{m^{2}}{2} \phi^{2}(x)\right), \\
H_{I} & =\int d x^{3}\left(\frac{\lambda}{4 !} \phi^{4}(x)+\frac{i \xi}{2 \sqrt{6 !}}\left\{\phi^{3}(x), \pi(x)\right\}\right),
\end{aligned}
$$

where $\phi$ is a one component scalar field, $\pi$ is its conjugate momenta and $\{A, B\}$ is the anticommutator of two operators $A$ and $B$. Also, $\epsilon$ is a parameter which can be set to one at the end. Note that $\phi$ and $\pi$ are satisfying the commutation relations $[\phi(x), \pi(y)]=i \delta^{3}(x-y)$ and $[\phi(x), \phi(y)]=[\pi(x), \pi(y)]=0$. 
The Hamiltonian model in Eq.(3) is non-Hermitian but according to Mostafazadeh, if there exists a positive definite metric operator $\eta$ such that $\eta H \eta^{-1}=H^{\dagger}$, then the spectrum of $H$ is real [14, 15]. Note also that, if $\eta$ exists then there exists an equivalent Hermitian Hamiltonian operator $h$ such that;

$$
h=\rho H \rho^{-1},
$$

where $\rho=\sqrt{\eta}$. In using the relation $\eta H \eta^{-1}=H^{\dagger}$ and employing the form $\eta=\exp (-Q)$ we get the result;

$$
\begin{aligned}
H^{\dagger} & =\int d y^{3}(\exp (-Q(y)) H(x) \exp (Q(y))) \\
& =\left(\begin{array}{c}
H(x)+\int d y^{3}[-Q(y), H(x)]+\frac{1}{2 !} \int d y^{3} \int d z^{3}[-Q(y),[-Q(z), H(x)]] \\
+\frac{1}{3 !} \int d y^{3} \int d z^{3} \int d \nu^{3}[-Q(y),[-Q(z),[-Q(\nu), H(x)]]]+\ldots
\end{array}\right),
\end{aligned}
$$

where

$$
Q(y)=Q_{0}(y)+\epsilon Q_{1}(y)+\epsilon^{2} Q_{2}(y)++\epsilon^{3} Q_{3}(y)+. .
$$

Explicitly we have;

$$
\begin{aligned}
& \exp (-Q) H \exp (Q)=H_{0}+\epsilon H_{I}+\left[-Q, H_{0}\right]+\left[-Q, \epsilon H_{I}\right]+\frac{1}{2 !}\left[-Q,\left[-Q, H_{0}\right]\right]+ \\
& \frac{1}{2 !}\left[-Q,\left[-Q, \epsilon H_{I}\right]\right]+\frac{1}{3 !}\left[-Q,\left[-Q,\left[-Q, H_{0}\right]\right]+\frac{1}{3 !}\left[-Q,\left[-Q,\left[-Q, \epsilon H_{I}\right]\right] \ldots\right.\right. \\
&=H_{0}+\epsilon H_{I}^{\dagger},
\end{aligned}
$$

and thus

$$
\begin{aligned}
0 & =\left[-Q_{0}, H_{0}\right] \Rightarrow Q_{0}=0 \text { is a good choice. } \\
H_{n} & =\frac{1}{2}\left[Q_{1}, H_{0}\right] \\
0 & =\left[-Q_{2}, H_{0}\right]+\left[-Q_{1}, H_{I}\right]+\frac{1}{2 !}\left[Q_{1},\left[Q_{1}, H_{0}\right]\right] \\
0 & =\left[-Q_{3}, H_{0}\right]+\left[-Q_{2}, H_{I}\right]+\frac{1}{2 !}\left[Q_{2},\left[Q_{1}, H_{0}\right]\right] \\
& +\frac{1}{2 !}\left[Q_{1},\left[Q_{2}, H_{0}\right]\right]+\frac{1}{3 !}\left[-Q_{1},\left[-Q_{1},\left[-Q_{1}, H_{0}\right]\right]\right] \\
& +\frac{1}{3 !}\left[-Q_{1},\left[-Q_{1}, H_{I}\right]\right]
\end{aligned}
$$

where $H_{n}$ is the non-Hermitian term in the interaction Hamiltonian $H_{I}$. To get a Hermitian representation for the model $H=H_{0}+\epsilon H_{I}$, one search for transformations which are able to kill the non-Hermitian interaction term $H_{I}$. In fact, the assumption that $Q(\phi)$ is a real 
functional of $\phi$ only will do the job because then the transformation of $H_{0}$ with a suitable choice of $Q(\phi)$ will result in another functional of $\phi$ times $\pi$. Considering this, one can expect a great simplification to the above set of coupled operator equations. To show this, consider the transformation of $H_{I}$;

$$
\exp (-Q) H_{I} \exp (Q)
$$

since $H_{I}$ is linear in $\pi$ then the commutators $\left[Q_{n}, H_{I}\right]$ are all functionals in $\phi$ only. Accordingly, the above set will take the form;

$$
\begin{aligned}
Q_{0} & =0, \\
H_{n} & =\frac{1}{2}\left[Q_{1}, H_{0}\right], \\
Q_{2} & =Q_{3}=Q_{4} \ldots .=0 .
\end{aligned}
$$

Now, $Q_{1}$ is an operator when commuted with $H_{0}(x)$ gives $2 \int d x^{3} \frac{i \xi}{\sqrt{6 !}}\left\{\phi^{3}(x), \pi(x)\right\}$, then one can expect $Q_{1}$ to take the form;

$$
Q_{1}=\int d y^{3} \frac{\xi \phi^{4}(y)}{2 \times \sqrt{6 !}}
$$

Thus,

$$
\eta=\exp \left(-\int d y^{3} \frac{\xi \phi^{4}(y)}{2 \times \sqrt{6 !}}\right)
$$

To obtain the equivalent Hermitian Hamiltonian consider the operator $\rho$ such that;

$$
\rho=\exp \left(-\int d y^{3} \frac{\xi \phi^{4}(y)}{4 \times \sqrt{6 !}}\right)=\exp \left(-\int d y^{3} \omega(y)\right),
$$

where we put $\omega(y)=\frac{\xi \phi^{4}(y)}{4 \times \sqrt{6 !}}$.

Then we have the form;

$\rho H(x) \rho^{-1}=H(x)+\int d y^{3}[-\omega(y), H(y)]+\frac{1}{2} \int d z^{3} \int d y^{3}[-\omega(z),[-\omega(y), H(x)]]+\ldots \ldots$ In fact, only terms which have $\pi$ will be effective in calculating the commutators and thus we have the result;

$$
\begin{aligned}
{\left[-\omega(y), \frac{\pi^{2}(x)}{2}\right] } & =[-\omega(y), \pi(x)] \frac{\pi(x)}{2}+\frac{\pi(x)}{2}[-\omega(y), \pi(x)] \\
& =-i \frac{\partial \omega(y)}{\partial \phi(y)} \frac{\pi(x)}{2} \delta^{3}(x-y)-i \frac{\pi(x)}{2} \frac{\partial \omega(y)}{\partial \phi(y)} \delta^{3}(x-y) \\
& =-\frac{1}{2} i \frac{\xi}{\sqrt{6 !}}\left\{\phi^{3}(y), \pi(x)\right\} \delta^{3}(x-y),
\end{aligned}
$$


and

$$
\begin{aligned}
{\left[-\omega(z),\left[-\omega(y), \frac{\pi^{2}(x)}{2}\right]\right] } & =\left[-\omega(z),-\frac{1}{2} i \frac{\xi}{\sqrt{6 !}}\left\{\phi^{3}(y), \pi(x)\right\} \delta^{3}(x-y)\right] \\
& =2 \frac{i}{2}\left(\frac{\xi}{\sqrt{6 !}} \phi^{3}(y)\right) i \frac{\partial \omega(z)}{\partial \phi(z)} \delta^{3}(x-y) \delta^{3}(x-z) \\
& =-\left(\frac{\xi}{\sqrt{6 !}} \phi^{3}(y)\right)^{2} \delta^{3}(x-y) \delta^{3}(x-z) .
\end{aligned}
$$

Then

$$
\rho\left(\int d^{3} x \frac{\pi^{2}(x)}{2}\right) \rho^{-1}=\int d^{3} x\left(\frac{\pi^{2}(x)}{2}-\frac{1}{2} i\left\{\frac{\xi}{\sqrt{6 !}} \phi^{3}(x), \pi(x)\right\}-\frac{1}{2}\left(\frac{\xi}{\sqrt{6 !}} \phi^{3}(x)\right)^{2}\right) .
$$

Similarly, we get

$$
\rho H_{I} \rho^{-1}=H_{I}+\int d y^{3}\left[-\omega(y), H_{n}(x)\right]
$$

Now we have;

$$
\begin{aligned}
{\left[-\omega(y), H_{n}(x)\right] } & =\left[-\omega(y), i \frac{\xi}{2 \sqrt{6 !}}\left\{\phi^{3}(x), \pi(x)\right\}\right] \\
& =2\left(i \frac{\xi}{2 \sqrt{6 !}} \phi^{3}(x)\right)[-\omega(y), \pi(x)] \\
& =2\left(i \frac{\xi}{2 \sqrt{6 !}} \phi^{3}(x)\right)\left(-i \frac{\partial \omega(y)}{\partial \phi(y)}\right) \delta^{3}(x-y) \\
& =\left(\frac{\xi}{\sqrt{6 !}} \phi^{3}(x)\right)^{2} \delta^{3}(x-y) .
\end{aligned}
$$

Collecting the different terms one gets the Hermitian Hamiltonian of the form;

$$
\begin{aligned}
h & =\int d x^{3}\left(\begin{array}{c}
\frac{1}{2}(\nabla \phi(x))^{2}+\frac{\pi^{2}(x)}{2}+\frac{1}{2} m^{2} \phi^{2}(x)+\frac{g}{4 !} \phi^{4}(x) \\
-\frac{1}{2}\left(\frac{\xi}{\sqrt{6 !}} \phi^{3}(x)\right)^{2}+\left(\frac{\xi}{\sqrt{6 !}} \phi^{3}(x)\right)^{2}
\end{array}\right) \\
& =\int d x^{3}\left(\frac{1}{2}(\nabla \phi(x))^{2}+\frac{\pi^{2}}{2}+\frac{1}{2} m^{2} \phi^{2}(x)+\frac{g}{4 !} \phi^{4}(x)+\frac{1}{2}\left(\frac{\xi}{\sqrt{6 !}} \phi^{3}(x)\right)^{2}\right) \\
& =\int d x^{3}\left(\frac{\pi^{2}(x)}{2}+\frac{1}{2}(\nabla \phi(x))^{2}+\frac{1}{2} m^{2} \phi^{2}(x)+\frac{g}{4 !} \phi^{4}(x)+\frac{1}{2}\left(\frac{\xi}{6 !}\right)^{2} \phi^{6}\right) .
\end{aligned}
$$

This form of the equivalent Hermitian Hamiltonian assures the real spectrum of the nonHermitian Hamiltonian model in Eq.(3). The most interesting realization is that the mass dimension of the couplings of the interaction Hamiltonians in both the equivalent models in Eqs.(3(13) are different. In Eq.(3), the coupling of the non-Hermitian interaction Hamiltonian $\xi$ has a mass dimension of the form $M^{-1}$ in $3+1$ dimensions, where $M$ is a mass 
unit. On the other hand, the Hermitian Hamiltonian in Eq.(13), the $\phi^{6}$ term has a coupling $\propto \xi^{2}$ which has a mass dimensions of -2 in $3+1$ dimensions. This is a very interesting result because the dimensionality of the coupling constant determines whether the theory is super-renormalizable ( coupling of positive mass dimension), renormalizable (dimensionless coupling) and non-renormalizable (coupling of negative mass dimension). However, our result showed that the coupling dimensions of some theory is representation dependent. Relying on this closed form result and the perturbative calculations in Ref .[12], one can legally aim to find a representation for a non-renormalizable theory in which the theory is renormalizable. This kind of future research is very interesting toward the unification of the four forces in our universe as gravity has a coupling of negative 2 mass dimension. Moreover, the $\phi^{6}$ theory which has a coupling of negative 2 mass dimension seems to be the only candidate to play a role in early universe studies to account for a strong first order phase transition needed for the matter-antimatter asymmetry in our universe. In view of our work and the results in Ref.[12], we think that non-renormalizability of a theory is not a conceptual but a technical problem that one can (in principle) get rid of it.

The non-Hermitian form studied above has another advantage. In fact, the $\phi^{6}$ theory is a very interesting quantum field model for critical phenomena studies and also for the search for models that have bound states. However, the quantum field calculations for this model is not easy as each vertex has an emergence of six lines and thus has a lengthy type of calculations. On the other hand, the equivalent non-Hermitian form in Eq.(3) is a $\phi^{4}$ - like theory with only four lines emerging at each vertex and thus the calculations in this representation goes more easily than in the Hermitian representation.

To make the above studies more general, we consider the scalar quantum field Hamiltonian of the form;

$$
H=\int d x^{3}\left(\frac{\pi^{2}(x)}{2}+\frac{1}{2}(\nabla \phi(x))^{2}+m \frac{\phi^{2}(x)}{2}+i\{G[\phi(x)], \pi(x)\}\right),
$$

where $G[\phi(x)]$ is a functional in the scalar field $\phi$. The form of $\eta_{+}$may be expected to have the form;

$$
\begin{aligned}
\eta_{+} & =\rho^{2} \\
\rho & =\int d y^{3} \exp (-\omega[\phi(y)]),
\end{aligned}
$$


where $\omega[\phi(x)]$ is a functional in the scalar field $\phi$ to be obtained. Using Baker-CampbellHausdorff formula we obtain,

$$
\rho H \rho^{-1}=H+\int d y^{3}[-\omega(y), H]+\int d y^{3} \int d z^{3} \frac{1}{2}[-\omega(z),[-\omega(y), H]]+\ldots \ldots
$$

Now

$$
\begin{aligned}
{\left[-\omega(y), \frac{\pi^{2}(x)}{2}\right] } & =[-\omega(y), \pi(x)] \frac{\pi(x)}{2}+\frac{\pi(x)}{2}[-\omega(y), \pi(x)], \\
& =\left(-i \frac{\partial \omega(y)}{\partial \phi(y)} \frac{\pi(x)}{2}-i \frac{\pi(x)}{2} \frac{\partial \omega(y)}{\partial \phi(y)}\right) \delta^{3}(x-y),
\end{aligned}
$$

and

$$
\begin{aligned}
{\left[-\omega(z),\left[-\omega(y), \frac{\pi^{2}(x)}{2}\right]\right] } & =\left[-\omega(z),-i\left(\frac{\partial \omega(y)}{\partial \phi(y)} \frac{\pi(x)}{2}+\frac{\pi(x)}{2} \frac{\partial \omega(y)}{\partial \phi(y)}\right)\right] \delta^{3}(x-y), \\
& =-\frac{1}{2}\left(\frac{\partial \omega(z)}{\partial \phi(z)} \frac{\partial \omega(y)}{\partial \phi(y)}+\frac{\partial \omega(z)}{\partial \phi(z)} \frac{\partial \omega(y)}{\partial \phi(y)}\right) \delta^{3}(x-z)(x-z) .
\end{aligned}
$$

Therefore,

$$
\left[-\omega(Q),\left[-\omega(z),\left[-\omega(y), \frac{\pi^{2}(x)}{2}\right]\right]\right]=0,
$$

and all the subsequent terms generated from the use of Baker-Campbell-Hausdorff formula used for the commutation of $\omega(Q)$ with $\frac{\pi^{2}(x)}{2}$ are zero too. In other words, the closed form transformation of $H_{0}$ yields

$$
\begin{aligned}
\rho H_{0} \rho^{-1} & =H_{0}+\int d^{3} x \int d^{3} y\left(-i \frac{\partial \omega(y)}{\partial \phi(y)} \frac{\pi(x)}{2}-i \frac{\pi(x)}{2} \frac{\partial \omega(y)}{\partial \phi(y)}\right) \delta^{3}(x-y) \\
& +\frac{1}{4} \int d^{3} x \int d^{3} y \int d^{3} z\left(-\frac{\partial \omega(z)}{\partial \phi(z)} \frac{\partial \omega(y)}{\partial \phi(y)}-\frac{\partial \omega(z)}{\partial \phi(z)} \frac{\partial \omega(y)}{\partial \phi(y)}\right) \delta^{3}(x-z) \delta^{3}(x-z) \\
& =H_{0}-\frac{1}{2} i \int d^{3} x\left\{\frac{\partial \omega(x)}{\partial \phi(x)}, \pi(x)\right\}-\frac{1}{2} \int d^{3} x\left(\frac{\partial \omega(x)}{\partial \phi(x)}\right)^{2} .
\end{aligned}
$$

Now let us consider the transformation of $H_{I}$ and noting that;

$$
\begin{aligned}
{[-\omega(y),\{i G[\phi(x)], \pi(x)\}] } & =-i([\omega(y), G[\phi(x)] \pi(x)]+[\omega(y), \pi(x) G[\phi(x)]]), \\
& =2 G[\phi(x)]\left(\frac{\partial \omega(y)}{\partial \phi(y)}\right) \delta^{3}(x-y),
\end{aligned}
$$

Then

$$
\begin{aligned}
\rho H_{I} \rho^{-1} & =H_{I}+2 \int d^{3} x \int d^{3} y G[\phi(x)]\left(\frac{\partial \omega(y)}{\partial \phi(y)}\right) \delta^{3}(x-y), \\
& =H_{I}+2 \int d^{3} x G[\phi(x)]\left(\frac{\partial \omega(x)}{\partial \phi(x)}\right)
\end{aligned}
$$


Accordingly,

$$
\begin{aligned}
\rho H \rho^{-1} & =h=H-\frac{1}{2} i \int d^{3} x\left\{\frac{\partial \omega(x)}{\partial \phi(x)}, \pi(x)\right\}-\frac{1}{2} \int d^{3} x\left(\frac{\partial \omega(x)}{\partial \phi(x)}\right)^{2} \\
& +2 \int d^{3} x G[\phi(x)]\left(\frac{\partial \omega(x)}{\partial \phi(x)}\right) \\
& =\int d x^{3}\left(\frac{\pi^{2}(x)}{2}+\frac{1}{2}(\nabla \phi(x))^{2}+m \frac{\phi^{2}(x)}{2}+i\{G[\phi(x)], \pi(x)\}\right), \\
& -\frac{1}{2} i \int d^{3} x\left\{\frac{\partial \omega(x)}{\partial \phi(x)}, \pi(x)\right\}-\frac{1}{2} \int d^{3} x\left(\frac{\partial \omega(x)}{\partial \phi(x)}\right)^{2} \\
& +2 \int d^{3} x G[\phi(x)]\left(\frac{\partial \omega(x)}{\partial \phi(x)}\right) .
\end{aligned}
$$

In fact, if $G[\phi(x)]=\frac{1}{2} \frac{\partial \omega(x)}{\partial \phi(x)}$, then $h$ is Hermitian and the operator $\eta=\rho^{2}$ serves as a positive definite metric operator. To check this, one uses the relation

$$
H^{\dagger}=\eta H \eta^{-1}=\rho \rho H \rho^{-1} \rho^{-1}=\rho h \rho^{-1} .
$$

Or

$$
\begin{aligned}
H^{\dagger} & =\rho h \rho^{-1}=\int d x^{3} \rho \frac{\pi^{2}(x)}{2} \rho^{-1}+\int d x^{3}\left(\frac{1}{2}(\nabla \phi(x))^{2}+m \frac{\phi^{2}(x)}{2}\right) \\
& -\frac{1}{2} \int d^{3} x\left(\frac{\partial \omega(x)}{\partial \phi(x)}\right)^{2}+2 \int d^{3} x G[\phi(x)]\left(\frac{\partial \omega(x)}{\partial \phi(x)}\right), \\
& =\int d x^{3}\left(\frac{\pi^{2}(x)}{2}+\frac{1}{2}(\nabla \phi(x))^{2}+m \frac{\phi^{2}(x)}{2}\right) \\
& -\frac{1}{2} i \int d^{3} x\left\{\frac{\partial \omega(x)}{\partial \phi(x)}, \pi(x)\right\}-\frac{1}{2} \int d^{3} x\left(\frac{\partial \omega(x)}{\partial \phi(x)}\right)^{2} \\
& -\frac{1}{2} \int d^{3} x\left(\frac{\partial \omega(x)}{\partial \phi(x)}\right)^{2}+2 \int d^{3} x G[\phi(x)]\left(\frac{\partial \omega(x)}{\partial \phi(x)}\right) \\
& =\int d x^{3}\left(\frac{1}{2}(\nabla \phi(x))^{2}+\frac{\pi^{2}(x)}{2}+m \frac{\phi^{2}(x)}{2}\right)-i \int d^{3} x\{G[\phi(x)], \pi(x)\} .
\end{aligned}
$$

Accordingly, the form $\eta=\int d y^{3} \exp (-\omega(y))$ passes all the tests as a positive definite metric operator for the pseudo Hermitian Hamiltonian of the scalar field theory of the form;

$$
H=\int d x^{3}\left(\frac{\pi^{2}(x)}{2}+\frac{1}{2}(\nabla \phi(x))^{2}+m \frac{\phi^{2}(x)}{2}+i\{G[\phi(x)], \pi(x)\}\right) .
$$

Let us now consider some specific choices for the functional $G[\phi(x)]$ :

\section{Case 1: $\phi^{4}$ equivalent}


In this case, the functionals $w[\phi(x)]$ and $G[\phi(x)]$ take the forms;

$$
\begin{aligned}
w[\phi(x)] & =\frac{g \phi^{3}(x)}{3}, \\
G[\phi(x)] & =\frac{1}{2} \frac{\partial \omega(x)}{\partial \phi(x)}=\frac{1}{2} g \phi^{2}(x) .
\end{aligned}
$$

then

$$
\begin{aligned}
h & =\int d x^{3}\left(\frac{\pi^{2}(x)}{2}+\frac{1}{2}(\nabla \phi(x))^{2}+m \frac{\phi^{2}(x)}{2}+\frac{1}{2} i g\left\{\left[\phi^{2}(x)\right], \pi(x)\right\}\right), \\
& -\frac{1}{2} i g \int d^{3} x\left\{\frac{1}{2} \phi^{2}(x), \pi(x)\right\}-\frac{1}{2} g^{2} \int d^{3} x\left(\phi^{2}(x)\right)^{2}+g^{2} \int d^{3} x \phi^{4}(x), \\
& =\int d x^{3}\left(\frac{\pi^{2}(x)}{2}+\frac{1}{2}(\nabla \phi(x))^{2}+m \frac{\phi^{2}(x)}{2}+\frac{1}{2} g^{2} \phi^{4}(x)\right) .
\end{aligned}
$$

\section{Case 2: $\phi^{6}$ equivalent}

In this case; we choose the functional $w[\phi(x)]$ as

$$
\begin{aligned}
w[\phi(x)] & =\frac{\lambda \phi^{4}(x)}{4}, \\
G[\phi(x)] & =\frac{1}{2} \frac{\partial \omega(x)}{\partial \phi(x)}=\frac{1}{2} \lambda \phi^{3}(x),
\end{aligned}
$$

and thus

$$
\begin{aligned}
h & =\int d x^{3}\left(\frac{\pi^{2}(x)}{2}+\frac{1}{2}(\nabla \phi(x))^{2}+m \frac{\phi^{2}(x)}{2}\right) \\
& -\frac{1}{2} \int d^{3} x\left(\lambda \phi^{3}(x)\right)^{2}+4 \int d^{3} x\left(\frac{\lambda \phi^{3}(x)}{2}\right)^{2} \\
& =\int d x^{3}\left(\frac{1}{2}(\nabla \phi(x))^{2}+\frac{(\pi(x))^{2}}{2}+m \frac{\phi^{2}(x)}{2}+\frac{1}{2} \lambda^{2} \phi^{6}(x)\right) .
\end{aligned}
$$

\section{Case 3: $\phi^{8}$ equivalent}

In this case we choose the functional $w[\phi(x)]$ as

$$
\begin{aligned}
& w[\phi(x)]=\frac{\lambda_{8} \phi^{4}(x)}{4}+\frac{\lambda_{6} \phi^{3}(x)}{3}+\frac{\lambda_{4} \phi^{2}(x)}{2}, \\
& G[\phi(x)]=\frac{1}{2} \frac{\partial \omega(x)}{\partial \phi(x)}=\frac{1}{2}\left(\lambda_{8} \phi^{4}(x)+\lambda_{6} \phi^{3}(x)+\lambda_{4} \phi^{2}(x)\right)
\end{aligned}
$$

and thus

$$
\begin{aligned}
h & =\int d x^{3}\left(\frac{\pi^{2}(x)}{2}+\frac{1}{2}(\nabla \phi(x))^{2}+m \frac{\phi^{2}(x)}{2}\right) \\
& +\frac{1}{2} \int d^{3} x\left(\lambda_{8}^{2} \phi^{8}+2 \lambda_{6} \lambda_{8} \phi^{7}+\left(\lambda_{6}^{2}+2 \lambda_{4} \lambda_{8}\right) \phi^{6}+2 \lambda_{4} \lambda_{6} \phi^{5}+\lambda_{4}^{2} \phi^{4}\right) .
\end{aligned}
$$


What is interesting in this representation is that it lowers the number of lines $n$ emerging from each vertex in the Hermitian theory to $\frac{n}{2}+1$ lines emerging from each vertex in the nonHermitian representation. This property lowers the number of available Feynman diagrams and thus simplifies the perturbative calculations. To elucidate this point, consider the two Feynman diagrams in Fig,1, which they correspond to second order (in the couplings) of the equivalent Hermitian $\phi^{4}$ theory and non-Hermitian $i \phi^{2} \pi$ theory. Both diagrams have the same number of vertices however the diagram that correspond to the non-Hermitian theory diverge as $\Lambda^{2 d-4}$ while the diagram that correspond to the Hermitian $\phi^{4}$ theory diverge as $\Lambda^{3 d-8}$. Moreover, in the non-Hermitian theory, one deals with three propagators while for the calculation of the diagram in the Hermitian theory one deals with four propagators. Accordingly, power counting shows that the UV behavior of the non-Hermitian theory is better as well as the Feynman diagrams calculations are simple provided that we compare diagrams of same number of vertices in the Hermitian and the equivalent non-Hermitian theories.

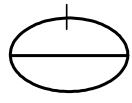

(a)

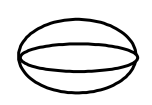

(b)

FIG. 1: Two Feynman diagrams with the same number of vertices in the Non-Hermitian theory $i\left\{\phi^{2}, \pi\right\}$ (diagram $(a)$ ) and the corresponding equivalent Hermitian $\phi^{4}$ theory (diagram $(b)$ ). The tick mark in one of the propagator of diagram $(a)$ refers to the contraction of two $\pi$ fields.

To conclude, we asserted the non-existence of a no go theorem for the possible physical acceptability of a non-renormalizable theory. To show that, we revisited a previous work by Carl M. Bender, Jun-Hua Chen, and Kimball A. Milton [12]. In this article, the au- 
thors found divergences in the Feynman diagrams generated by the Hermitian Hamiltonian. The importance of this result has been overlooked in the Benders et.al work as well as in the literature. Although the calculations in Ref.[12] have been done in $0+1$ space-time dimensions, we used dimensional analysis to show that the divergences found are due to superrenormalizable, renormalizable and non-renormalizable interaction terms in the Hermitian Hamiltonian. Since the Hermitian Hamiltonian is now non-renormalizable while the equivalent non-Hermitian theory is finite, we concluded the possibility of the dependence of the superficial degree of divergence of a theory on its representation (e.g Hermitian and non-Hermitian equivalent representations ). To elucidate the idea, we introduced a class of non-Hermitian Hamiltonians and obtained the equivalent class of Hermitian Hamiltonians in a closed form. For a non-Hermitian Hamiltonian in the class that has a coupling of mass dimension like $M^{\frac{\delta}{2}}$, we realized that the corresponding Hermitian Hamiltonian has a coupling of mass dimension as $M^{\delta}$. Accordingly, we proved the possibility of having two equivalent theories which have different superficial degree of divergences. This is a very interesting result which may help in finding a solution to the unification problem.

The Hermitian representation is of the form $\phi^{2 n}$ scalar field theory while the equivalent non-Hermitian representation is a $\phi^{n+1}$-like theory which turn the calculations more simpler in this representation than in the Hermitian one. Besides, the metric operator is supposed to disappear from path integral calculations which mean that physical amplitudes can be fully obtained in the simpler non-Hermitian representation.

[1] Frieder Kleefeld, J. Phys. A: Math. Gen. 39 L9-L15 (2006).

[2] Symanzik K, Springer Tracts Mod. Phys. 57 222-36 (1971).

[3] Symanzik K, Commun. Math. Phys. 23 49-86 (1971).

[4] Symanzik K, Nuovo Cimento 6 77-80 (1973).

[5] Carl Bender and Stefan Boettcher, Phys.Rev.Lett.80:5243-5246 (1998).

[6] Abouzeid Shalaby and Suleiman S. Al-Thoyaib, Phys. Rev.D 82, 085013 (2010)

[7] Abouzeid M. Shalaby, Phys.Rev.D80:025006 (2009).

[8] Carl M. Bender and Philip D. Mannheim, Phys.Rev.Lett.100:110402 (2008).

[9] Carl M. Bender, Sebastian F. Brandt, Jun-Hua Chen and Qinghai Wang, Phys.Rev. D71, 
025014 (2005).

[10] Edward W.Kolb and Michaels. Turner, The Early Universe, ADDISON-WESLEY PUBLISHING COMPANY, (1990).

[11] Christophe Grojean, Geraldine Servant and James D. Wells, Phys.Rev.D71:036001 (2005).

[12] Carl M. Bender, Jun-Hua Chen, and Kimball A. Milton, J.Phys.A39:1657-1668 (2006).

[13] Michael E. Peskin and Daniel V.Schroeder, An Introduction To Quantum Field Theory (Addison-Wesley Advanced Book Program) ( 1995).

[14] A. Mostafazadeh, J. Math. Phys., 43, 3944 (2002).

[15] A. Mostafazadeh, J. Math. Phys. 43, 205 (2002).

[16] In fact, it is a Hierarchy problem too. 